\title{
Biosynthesis of Lipids during Embryogenesis of Ascaris lumbricoides Eggs
}

\author{
Yuji Furukawa, Tokuo Yamamoto, Shuichi Kimura, \\ Hisako Hayashi* and Hiroshi Oya* \\ Laboratory of Nutrition, Department of Food Chemistry, \\ Faculty of Agriculture, Tohoku University, Sendai 980 \\ and ${ }^{*}$ School of Medicine, Juntendo University, Tokyo 113
}

\begin{abstract}
Furukawa, Y., Yamamoto, T., Kimura, S., Hayashi, H. and Oya, H. Biosynthesis of Lipids During Embryogenesis of Ascaris lumbricoides Eggs. Tohoku J. exp. Med., 1985, 147 (3), 221-234 — Alteration in lipid metabolism of Ascaris lumbricoides eggs during development in either air or nitrogen gas was studied using acetate- $1-{ }^{14} \mathrm{C}$ and labeled fatty acids. It is thought that formation of ${ }^{14} \mathrm{C}$-lipids and ${ }^{14} \mathrm{C}$-palmitoleate from acetate- $1-{ }^{14} \mathrm{C}$ and palmitate- $1-{ }^{14} \mathrm{C}$ in developing eggs may be influenced by the concentration of molecular oxygen in the medium. Acetate- $1-{ }^{-14} \mathrm{C}$ was incorporated into palmitate, palmitoleate and unsaturated 18-carbon acid of fatty acids in the eggs, but the incorporation into saturated 18-carbon acid was slight. Radioactive fatty acid methyl esters were majer lipid component of the radioactive lipid classes in the first and second stage larvae incubated with palmitate- $1-{ }^{14} \mathrm{C}$. Isopentadecanoic acid, which is a small component of fatty acids in Ascaris eggs, contains a high percentage of the total radioactivity in the second stage larvae incubated with acetate- $1-{ }^{14} \mathrm{C}$ under both air and nitrogen. It appears that a conversion of the fatty acid biosynthesis system may occur during development in to the second stage larvae.

Ascaris lumbricoides; developing egg; fatty acid biosynthesis ; isopentadecanoic acid
\end{abstract}

It is well known that the adult worm of Ascaris lumbricoides lives in predominantly anaerobic environments, such as the intestine, whereas the larvae live for some time in aerobic environments, such as the lung. Von Brand (1952) suggested that fatty acid synthesis in essentially anaerobic parasites serves to oxidize reduced metabolic substrates. The synthesized fats of the adult are stored in the eggs and larvae where they may be utilized as an energy source in development (Fairbairn 1960). The general characteristics and metabolism of the lipids of Ascaris (Beames et al. 1967) and triglyceride utilization or the conversion of triglycerides to carbohydrates in devoloping eggs of Ascaris lumbricoides are known in some detail (Jezyk and Fairbairn 1969: Barrett et al. 1970), but

Received March 18, 1985; accepted for publication July 26, 1985. 
information on the fatty acid biosynthesis of the larvae is quite limited. The present report deals with changes in the ability to synthesize lipids and fatty acid biosynthesis during the embryonation in Ascaris lumbricoides eggs.

\section{Materials and Methods}

Materials. Sodium acetic acid- $1-^{14} \mathrm{C}$, palmitic acid- $1-{ }^{14} \mathrm{C}$ were purchased from New England Nuclear, Inc. Silicic acid (100 mesh) and silica gel G (Type 60) were obtained from Mallinckrodt Chemical Works and Merk, respectively.

Preparation of eggs. Eggs were removed from the lower third of the uteri of mature female Ascaris obtained from a local slaughterhouse, suspended in $0.5 \mathrm{~N} \mathrm{NaOH}$ overnight to remove the outer protein coat, lightly homogenated with a Potter-Elvehjem type homogenizer, and washed with distilled water. The eggs were also washed with n-butanol to separate the unfertilized eggs. These fertilized eggs were washed again with distilled water, and maintained in $0.1 \mathrm{~N}$ sulfuric acid at $5^{\circ} \mathrm{C}$ until needed (undeveloped fertilized eggs-A) (Fairbairn 1955). The eggs suspension in $30 \mathrm{ml}$ aliquots was incubated with air bubbling at $30^{\circ} \mathrm{C}$ for 7 days (vermiform eggs-B), 14 days (first stage larvae-C) and 21 days (second stage larvae-D), respectively.

Preparation of ${ }^{14} \mathrm{C}$-fatty acid-albumin complex. One hundred microliters $(100 \mu \mathrm{Ci})$ of benzen solution of palmitic acid-1- ${ }^{14} \mathrm{C}$ with a specific activity of $53.2 \mathrm{mCi} / \mathrm{mmole}$ in a polypropylene tube were dried under nitrogen. Ten milliliters of $0.25 \mu$ mole bovine serum albumin in $50 \mathrm{mM}$ Tris buffer, $\mathrm{pH} 7.4$ was added to the tube. The mixture was sonicated with Kubota KMS-100 sonifier at $10 \mathrm{kHz}$ for $10 \mathrm{~min}$. One milliliter of the sonicated suspension contained a $10 \mu \mathrm{Ci}$ of palmitic acid-1-14 $\mathrm{C}$.

Incubation of the larvae with acetate $-1-^{-14} \mathrm{C}$ or labeled fatty acid. Before the incubation with labeled fatty acid, eggs of the four stages were sonicated individually. One $\mathrm{ml}$ of the sonicated egg suspension (containing 300 to $600 \mathrm{mg}$ eggs as dry weight) was incubated with $1 \mathrm{ml}$ of acetate- $1{ }^{14} \mathrm{C}(20 \mu \mathrm{Ci})$ or the sonicated bovine albumin- ${ }^{14} \mathrm{C}$-fatty acid complex (10 $\mu \mathrm{Ci}$ ) in $5 \mathrm{ml}$ of $50 \mathrm{mM}$ Tris buffer, $\mathrm{pH} 7.4$, as a final volume. Each tube was continously bubbled with air or nitrogen gas $(10 \mathrm{ml} / \mathrm{min})$ and maintained at $30^{\circ} \mathrm{C}$ for $24 \mathrm{hr}$. Commercial preparations of nitrogen gas contain small quantities of oxygen (Saz and Lescure. 1966). Therefore, it may not be said that the nitrogen bubbling method provides a strictly anaerobic condition for the eggs.

Extraction and identification of lipids. The harvested eggs were washed three times with cold saline, and stored overnight in a deep freezer prior to the extraction of lipids. The eggs were treated with trichloro-acetic acid (final concentration 5\%). The treated eggs were collected by centrifugation and suspended in $100 \mathrm{ml}$ of methanol. The methanol suspension was heated in a sealed tube under nitrogen gas at $55^{\circ} \mathrm{C}$ for $15 \mathrm{~min}$. After it was cooled, $200 \mathrm{ml}$ of chloroform was added and the solution was agitated for $24 \mathrm{hr}$ at $4^{\circ} \mathrm{C}$. The extract was then filtrated through a glass filter, and washed three times with an equal volume of $2 \mathrm{M} \mathrm{KCl}$ and then with distilled water. The lipids were fractionated by thinlayer chromatography with the use of a solvent system containing petroleum ether: ethylether: glacial acetic acid $(82: 18: 1, \mathrm{v} / \mathrm{v})$. After being dried, the plates were developed with iodine vapour, and radioautography was carried out. The relative amounts of each lipid class were calculated by a thin-layer chromatogram scanner (Aloka) by tracing the chromatography recordings on heavy paper and cutting out the individual peaks and weighting them. The extracted lipids were methylated with $\mathrm{BF}_{3}$-methanol (Metcalfe and Schmitz 1961). The various methylester fractions were subjected to gas liquid chromatography (Yanaco gas chromatograph model G 80 equipped with TCD detector) with a stainless steel column containing $15 \%$ diethylene glycol succinate polyester on 80-100 mesh chromosorb W (Nihon Chromato Industry Co., Ltd.). The operating conditions were: column temperature; $195^{\circ} \mathrm{C}$, carrier gas ; helium, carrier gas flow rate; $25 \mathrm{ml} / \mathrm{min}$. Fatty 
acid fractions which effluented were collected in a cooled $U$ tube attached to the heated outlet of the instrument and eluted with $10 \mathrm{ml}$ of toluene scintillator and counted with a Packard liquid scintillation counter, model 3385. Individual fatty acids were identified by comparing their relative retention times with those of known standards.

\section{Results}

Incorporation of acetate $-1-{ }^{14} \mathrm{C}$ and palmitate $-1-{ }^{14} \mathrm{C}$ into lipid fractions and its fatty acids

Table 1 shows the incorporation of acetate- $1-{ }^{14} \mathrm{C}$ and palmitate- $1-{ }^{14} \mathrm{C}$ into lipid fractions of eggs of all four stages under air and nitrogen gas. In the case of acetate- $1-{ }^{14} \mathrm{C}$, the amounts of ${ }^{14} \mathrm{C}$-lipid formed in the eggs of all stages under air were 6 to 190-times higher than those under nitrogen. This may indicate that lipid synthesis from acetate in these eggs was influenced by the concentration of molecular oxygen in the medium. In contrast to acetate- $1-{ }^{14} \mathrm{C}$, the incorporation of palmitate- $1-{ }^{14} \mathrm{C}$ into lipid fractions under nitrogen was considerably higher than that under air in the eggs of all stages. It thus appears that the palmitate incorporated into the eggs is highly susceptible to oxidation under air. In other words, since molecular oxygen is an essential requirement for the development of Ascaris eggs (Passey and Fairbairn 1955; Saz and Lescure 1965, 1966; Barrett et al. 1970), the eggs might utilize certain fatty acids as an energy source during the development under these conditions (Jezyk and Fairbairn 1969).

The distribution of radioactivity in each lipid class of the lipid fraction of the eggs incubated with acetate- $1-{ }^{14} \mathrm{C}$ under air is shown in Table 2. A survey of the

TABLE 1. Effect of gas phase on incorporation of acetate- $1 \mathrm{-}^{14} \mathrm{C}$ and palmitate- $1{ }^{14} \mathrm{C}$ into lipid fractions of Ascaris eggs in developing stages

\begin{tabular}{cccccc}
\hline \multirow{2}{*}{ Stage of eggs } & \multicolumn{3}{c}{ Radioactivity $\left(\mathrm{cpm} \times 10^{4} / \mathrm{mg}\right.$ dry wt. $)$} \\
\cline { 2 - 3 } \cline { 2 - 3 } \cline { 5 - 6 } & \multicolumn{2}{c}{ Acetate-1-14 $\mathrm{C}$} & & \multicolumn{2}{c}{ Palmitate-1-1 ${ }^{14} \mathrm{C}$} \\
\cline { 2 - 3 } A & 32.6 & 0.5 & & 264.3 & 362.3 \\
B & 56.9 & 0.3 & & 91.0 & 134.0 \\
C & 7.5 & 1.2 & & 212.0 & 253.6 \\
D & 108.3 & 8.9 & & 195.4 & 325.3 \\
\hline
\end{tabular}

$0.6 \mathrm{~g}$ of 0 -day eggs, $0.26 \mathrm{~g}$ of 7-day eggs, $0.44 \mathrm{~g}$ of 14-day eggs and $0.43 \mathrm{~g}$ of 21 -day eggs, respectively (all expressed as dry

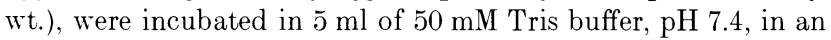
incubation flask with a sharpened bottom containing $20 \mu \mathrm{Ci}$ of acetate- $1-{ }^{14} \mathrm{C}$ or $10 \mu \mathrm{Ci}$ of palmitate- $1-{ }^{14} \mathrm{C}$ at $30^{\circ} \mathrm{C}$ under air and nitrogen. After $24 \mathrm{hr}$, the eggs were collected by centrifugation and the lipids were extracted with chloroform-methanol. 
TABLE 2. Distribution of radioactivity in lipid fractions of Ascaris eggs incubated with acetate $-1-{ }^{14} C$ under air

\begin{tabular}{lccc}
\hline & \multicolumn{3}{c}{ Relative radioactivity (\% total) } \\
\cline { 2 - 4 } & A & B & C \\
\hline Phospholipids & 92.7 & 89.9 & 34.4 \\
$\begin{array}{l}\text { Sterol, mono- and di-glycerides } \\
\text { Free fatty acids }\end{array}$ & - & - & - \\
Triglycerides & 7.3 & 10.1 & 30.9 \\
Unknown lipids & - & - & - \\
Sterol esters & - & - & 22.4 \\
\hline
\end{tabular}

Lipids from ascaris eggs ( $\mathrm{A}, \mathrm{B}$ and $\mathrm{D}$ of the developing stage) incubated with $20 \mu \mathrm{Ci}$ of acetate-1-14 $\mathrm{C}$ were fractionated by silicic acid thin-layer chromatography. The relative radioactivity of each lipid class was calculated from a tracing chart of a radio-chromatogram scanner. The solvent system and identification of lipids are described in the text.

TABLE 3. Distribution of radioactivity in lipid fractions of Ascaris eggs incubated with palmitate $-1{ }^{14} \mathrm{C}$

\begin{tabular}{|c|c|c|c|c|c|c|c|c|}
\hline \multirow{3}{*}{ Lipid fraction } & \multicolumn{8}{|c|}{ Relative radioactivity ( $\%$ total) } \\
\hline & \multicolumn{4}{|c|}{ Air } & \multicolumn{4}{|c|}{$\mathrm{N}_{2}$} \\
\hline & $\mathrm{A}$ & $\mathrm{B}$ & $\mathrm{C}$ & $\mathrm{D}$ & $\mathrm{A}$ & B & $\mathrm{C}$ & $\mathrm{D}$ \\
\hline Phospholipids & 7.4 & 19.5 & 19.8 & 12.3 & 3.0 & 21.1 & 13.9 & 13.0 \\
\hline $\begin{array}{l}\text { Sterol, mono- and } \\
\text { di-glycerides }\end{array}$ & - & 6.4 & 4.9 & 5.3 & - & 7.9 & 4.3 & 7.3 \\
\hline Free fatty acids & 85.7 & 46.4 & 37.2 & 30.3 & 81.6 & 48.5 & 25.8 & 25.0 \\
\hline Triglycerides & - & 19.8 & 12.5 & 18.1 & - & 12.3 & 14.5 & 24.7 \\
\hline Unknown lipids & 6.9 & 7.9 & 25.6 & 34.0 & 15.5 & 10.2 & 41.4 & 29.7 \\
\hline Sterol esters & - & - & - & - & - & - & - & 0.9 \\
\hline
\end{tabular}

Lipids from eggs of all four stages incubated with $10 \mu \mathrm{Ci}$ of palmitate-1-14 $\mathrm{C}$ were fractionated by silicic acid thin-layer chromatography. The solvent system, identification of lipids and calculation of the relative radioactivity of each lipid class are described in the text.

distribution of radioactivity incorporated into each lipid class under nitrogen and stage $\mathrm{C}$ under air was impossible due to the low radioactivity. It was observed that acetate- $1-{ }^{14} \mathrm{C}$ was mainly incorporated into phospholipids and fatty acids in unembryonated (A) and vermiform (B) eggs, respectively. On the other hand, in second stage larvae (D), much radioactivity was detected in an unknown lipid fraction $(22 \%)$ other than phospholipids $(34 \%)$ and fatty acids $(31 \%)$. These results suggest that a change in the lipid synthesis occured in this parasite during development under air. The distribution of radioactivity in each lipid class of the lipid fraction of the eggs incubated with palmitate- $1-{ }^{14} \mathrm{C}$ under air or nitrogen 
is shown in Table 3. In both atmospheres, unembryonated eggs (A) converted a small amount of palmitate to other lipid fractions, but upon more advanced development (B, C, D), such conversion gradually inereased and the relative radioactivities of triglycerides and unknown lipids increased. Indeed, the relative radioactivity of the ${ }^{14} \mathrm{C}$-fatty acid fraction decreased with the progression of development. It is suggested that the fatty acid metabolism in the larvae becomes more active with age.

It is also interesting that the unknown lipid was formed vigorously from both exogenously supplied acetate and palmitate in larvae of both first (C) and second (D) stages (Tables 2 and 3). It is therefore necessary to identify the neutral lipid fraction which was found to move between triglycerides and sterol esters on thin-layer chromatography, as illustrated in Fig. 1. Although the concentration of this unknown lipid was small in the parasite, much radioactivity was detected in this lipid fraction of the eggs incubated with both exogenously supplied

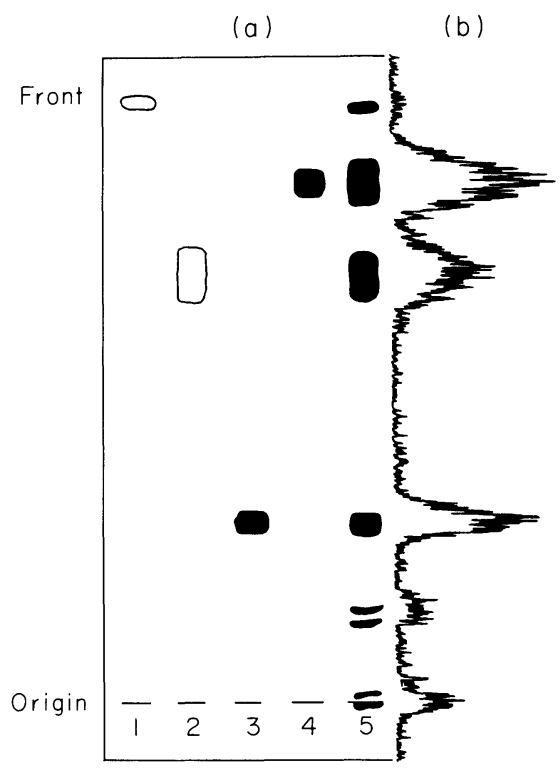

Fig. 1. Thin-layer chromatogram of total lipid extracted from Ascaris second stage larvae incubated with palmitate- $1-{ }^{-14} \mathrm{C}$ under nitrogen and its radioactive profile obtained from a radio-chromatogram scanner. Part (a) represents a thin-layer chromatogram of cholesterol-palmitate (1), trioleyl glyceride (2), palmitate- $1-{ }^{14} \mathrm{C}(3), 1-{ }^{14} \mathrm{C}$-palmitate methylester (4) and total lipid of Ascaris larvae incubated with palmitate- $1-{ }^{14} \mathrm{C}(5)$, respectively. Glass plate $(20 \times 20$ $\mathrm{cm}$ ) was coated with silica gel-G (Type 60, Merck) to a thickness of $0.5 \mathrm{~mm}$. The developing solvent system for the neutral lipid was petroleum ether: ethyl ether: acetic acid $(82: 18: 1, \mathrm{v} / \mathrm{v})$. At room temperature the chromatogram took about $1 \mathrm{hr}$ to run. After being dried, the plate was developed with iodine vapor (spots 1 and 2) and autoradiography was carried out (spots 3 and 4, and sample 5). Part (b) represents a distribution of radioactive lipids of sample 5 obtained with a radio-chromatogram scanner. 
TABLE 4. Distribution of radioactive fatty acid methyl esters in unidentified lipid fractions of Ascaris second stage larvae incubated with acetate $-1-{ }^{14} C$ under air

\begin{tabular}{cc}
\hline Fatty acid & $\begin{array}{c}\text { Relative radioactivity } \\
(\% \text { total })\end{array}$ \\
\hline$<14: 0$ (a) & 0.3 \\
$14: 0$ & 3.0 \\
$15: 0$ branched (b) & 31.0 \\
$15: 0,15: 1$ & 9.0 \\
$16: 0$ & 30.0 \\
$16: 1$ & 18.1 \\
$17: 0$ & 2.0 \\
$17: 1$ & 2.5 \\
$18: 0$ & 1.0 \\
$18: 0$ & 12.5 \\
\hline
\end{tabular}

A radioactive unidentified lipid fraction of total ${ }^{14} \mathrm{C}$-lipid from eggs incubated with acetate- $1-{ }^{14} \mathrm{C}$ was eluted with chloroform-methanol from a TLC plate and directly subjected to gas liquid chromatography without methylation.

Individual fatty acid peaks were collected in cooled U-shaped tubes, eluted with $10 \mathrm{ml}$ of toluene scintillator and counted with a liquid scintillation counter. (a) The numerals following the colon refer to the number of double bonds in the molecule, whereas the numerals before the colon refer to the number of carbon atoms of the fatty acid.

(b) Isobranched fatty acid.

acetate- $1-{ }^{14} \mathrm{C}$ and palmitate- $1-{ }^{14} \mathrm{C}$. To identify the unknown lipid, after extraction of the ${ }^{14} \mathrm{C}$-lipid fraction derived from acetate- $1-{ }^{14} \mathrm{C}$ on a TLC plate, the lipid fraction was subjected to GLC without methylation. A normal pattern of ${ }^{14} \mathrm{C}$-fatty acids was obtained qualitatively as shown in Table 4 . Furthermore, the mixture of the radioactive fatty acids did not have any unusual fatty acids and the distribution was similar to that of ${ }^{14} \mathrm{C}$-fatty acids of the total lipid fraction of the larvae (Table 5). These results suggest that the unidentified lipids are a mixture of fatty acid methyl esters.

\section{Studies of biosynthesis of fatty acids in Ascaris larvae}

The acetate- $1-{ }^{14} \mathrm{C}$ incorporation into fatty acids of the total lipid fraction is shown in Table 5. Among fatty acids of the total lipids the saturated 16 carbon acid contains a high percentage of radioactivity under both air and nitrogen. It has been suggested that the eggs (larvae) have a system of fatty acid synthesis by the malonyl-CoA pathway of vertebrates (Wakil et al. 1957). However, the low concentrations of radioactivity in the saturated 18 carbon acid $(0-1.3 \%)$ suggest 
that the rate of stearate synthesis in Ascaris eggs may be quite low. These results indicate that the parasite may not synthesize stearate by chain elongation from palmitate. This speculation is supported by the results, as shown in Table 6, which indicate a low incorporation $(0-0.5 \%)$ of the radioactivity from palmitate- $1-{ }^{14} \mathrm{C}$ into saturated 18 carbon acid.

The production of unsaturated carbon acid, which is generally formed by the desaturation of palmitate, was greatly influenced by the gas phase of the incubation medium. About 15 to $27 \%$ of the ${ }^{14} \mathrm{C}$-fatty acids produced from acetate-1${ }^{14} \mathrm{C}$ were detected in unsaturated 16 carbon acid under air (Table 5). These values were 2 to 3 times higher in relative radioactivity than those in first (C) and second (D) stage larvae of the parasite under nitrogen. These results suggest that the parasite produces the unsaturated 16 carbon acid by desaturation depending upon the concentration of molecular oxygen. The ratios of the conversion to ${ }^{14} \mathrm{C}$-unsaturated 16 carbon acid from palmitate- $1{ }^{-14} \mathrm{C}$ in second stage larvae were $16.7 \%$ and $2.0 \%$, as determined by relative radioactivity under air and nitrogen, respectively (Table 6). On the other hand, the formation of ${ }^{14} \mathrm{C}$-unsaturated 18 carbon acid from acetate- $1-{ }^{14} \mathrm{C}$ under air was considerably greater than that under nitrogen (Table 5). Notably, the radioactivity in the fatty acid was $53 \%$ of the total ${ }^{14} \mathrm{C}$-fatty acids in the case of first stage larvae. However, in light of the low radioactivity incorporated into saturated 18 carbon acid from acetate- $1-{ }^{14} \mathrm{C}$, the

TABLE 5. Acetate-1 $1{ }^{14} \mathrm{C}$ incorporation into fatty acids of eggs at each stage incubated under air and nitrogen

\begin{tabular}{|c|c|c|c|c|c|c|}
\hline \multirow{3}{*}{ Fatty acid } & \multicolumn{6}{|c|}{ Relative radioactivity ( $\%$ total) } \\
\hline & \multicolumn{4}{|c|}{ Air } & \multicolumn{2}{|c|}{$\mathrm{N}_{2}$} \\
\hline & A & B & $\mathrm{C}$ & $\mathrm{D}$ & $\mathrm{C}$ & $\mathrm{D}$ \\
\hline$<14: 0$ & - & 0.4 & 0.0 & 3.4 & 0.0 & 0.8 \\
\hline $14: 0$ & 1.0 & 1.2 & 0.7 & 2.1 & 1.8 & 9.7 \\
\hline $15: 0 \mathrm{br}$ & 7.4 & 5.4 & 0.5 & 33.4 & 8.2 & 36.1 \\
\hline $15: 0,1$ & 1.6 & 1.3 & 0.0 & 7.1 & 0.7 & 5.5 \\
\hline $16: 0$ & 34.0 & 32.9 & 20.6 & 16.6 & 76.5 & 24.8 \\
\hline $16: 1$ & 26.8 & 26.7 & 15.5 & 20.0 & 6.7 & 11.9 \\
\hline $17: 0,1$ & 0.2 & 4.7 & 2.4 & 5.0 & 0.4 & 3.1 \\
\hline $18: 0$ & 0.0 & 0.4 & 1.3 & 1.2 & 2.0 & 0.9 \\
\hline $18: 1$ & 29.1 & 24.6 & 53.4 & 9.7 & 0.8 & 4.3 \\
\hline $18: 2$ & 0.0 & 1.7 & 3.7 & 1.0 & 0.3 & 0.6 \\
\hline $18: 3$ & 0.0 & 0.0 & 0.4 & 0.3 & 0.0 & 0.6 \\
\hline $20: 0,1$ & 0.0 & 0.7 & 1.6 & 0.0 & 2.7 & 1.1 \\
\hline $20: 2$ & 0.0 & 0.0 & 0.0 & 0.0 & 0.0 & 0.3 \\
\hline $20: 4$ & 0.0 & 0.0 & 0.0 & 0.1 & 0.0 & 0.1 \\
\hline
\end{tabular}

The lipid fractions of Ascaris lumbricoides eggs incubated with acetate- $1-{ }^{14} \mathrm{C}$ were saponified and the fatty acids were extracted and analyzed as described in the text. 
TaBle 6. Distribution of radioactivity into major fatty acid of $A$. lumbricoides larvae incubated with palmitate- $1-{ }^{14} C$ under air and nitrogen

\begin{tabular}{lrrrrr}
\hline & \multicolumn{3}{c}{ Relative radioactivity (\% total) } \\
\cline { 2 - 3 } Fatty acid & \multicolumn{2}{c}{ Air } & & \multicolumn{2}{c}{$\mathrm{N}_{2}$} \\
\cline { 2 - 3 } \cline { 5 - 6 } & Stage C & Stage D & & Stage C & Stage D \\
\hline $15: 0 \mathrm{br}$ & 0.0 & 2.6 & & 0.0 & 0.1 \\
$16: 0$ & 95.3 & 80.3 & & 99.1 & 97.9 \\
$16: 1$ & 4.1 & 16.7 & & 0.9 & 2.0 \\
$18: 0$ & 0.5 & 0.0 & & 0.0 & 0.0 \\
$18: 1$ & 0.0 & 0.0 & & 0.0 & 0.0 \\
\hline
\end{tabular}

The lipid fractions of Ascaris lumbricoides larvae incubated with $10 \mu \mathrm{Ci}$ of palmitate- $1-{ }^{14} \mathrm{C}$ under air and nitrogen were saponified and the fatty acids were extracted and analyzed as described in the text.

TABLE 7. Distribution of major ${ }^{14} \mathrm{C}$-fatty acids in lipid fractions of the second stage larvae incubated with stearic acid $-1-^{-14} \mathrm{C}$

\begin{tabular}{ccc}
\hline \multirow{2}{*}{ Fatty acid } & \multicolumn{2}{c}{${ }^{14} \mathrm{C}$-fatty acid distribution $(\%)$} \\
\cline { 2 - 3 } & Air & $\mathrm{N}_{2}$ \\
\hline$<18: 0$ & 5.0 & 0.0 \\
$18: 0$ & 91.0 & 100.0 \\
$18: 1$ & 3.8 & 0.0 \\
$>18: 1$ & 0.0 & 0.0 \\
\hline
\end{tabular}

Lipids from eggs of the second stage larvae incubated with $10 \mu \mathrm{Ci}$ of stearic acid-1-14 $\mathrm{C}$ under air and nitrogen were saponified and the fatty acids were extracted and analyzed as described in the text.

formation of ${ }^{14} \mathrm{C}$-unsaturated 18 carbon acid does not seem to be the result of observable desaturation of saturated 18 carbon acid. Furthermore, ${ }^{14} \mathrm{C}$ unsaturated 18 carbon acid detected in the lipid fraction of the second stage larvae incubated with stearate- $1-{ }^{14} \mathrm{C}$ was quite low under both air and nitrogen (Table 7). Since the quantity of ${ }^{14} \mathrm{C}$-unsaturated 18 carbon acid formed from acetate- $1-{ }^{14} \mathrm{C}$ under air was larger than that formed under nitrogen (Table 5), the ${ }^{14} \mathrm{C}$ unsaturated fatty acid is not produced by an anaerobic desaturation process of unsaturated 16 carbon acid, palmitoleic acid (Stumpf 1962). It is likely that in developing eggs of Ascaris, the ${ }^{14} \mathrm{C}$-unsaturated 18 carbon acid is formed from a specific pathway depending upon molecular oxygen via certain precursors other than stearate and palmitoleate.

On the other hand, acetate- $1-{ }^{14} \mathrm{C}$ incorporation into branched 15 -carbon fatty acid, which is a minor component of fatty acids in the second stage larvae, is much 
TABLE 8. Major fatty acid composition of Ascaris eggs in developing stages

\begin{tabular}{lrrrr}
\hline & \multicolumn{4}{c}{ Relative amounts $(\%)$} \\
\cline { 2 - 5 } Fatty acid & \multicolumn{1}{c}{$\mathrm{A}$} & \multicolumn{1}{c}{$\mathrm{B}$} & $\mathrm{C}$ & \multicolumn{1}{c}{$\mathrm{D}$} \\
\hline $14: 0$ & 1.1 & 0.8 & 0.9 & 0.8 \\
$15: 0 \mathrm{br}$ & 2.0 & 1.6 & 1.9 & 1.9 \\
$15: 0$ & 0.3 & 0.2 & 0.2 & 0.3 \\
$16: 0$ & 17.5 & 15.1 & 15.1 & 11.9 \\
$16: 1$ & 0.9 & 1.7 & 2.3 & 2.1 \\
$17: 0$ & 0.5 & 0.5 & 0.4 & 0.4 \\
$17: 1$ & 0.2 & 0.4 & 0.4 & 1.2 \\
$18: 0$ & 10.0 & 9.3 & 10.8 & 10.5 \\
$18: 1$ & 30.1 & 32.1 & 35.4 & 33.5 \\
$18: 2$ & 30.7 & 30.7 & 27.0 & 28.8 \\
$18: 3$ & 1.2 & 0.6 & 1.3 & 0.2 \\
$20: 0$ & 0.4 & 0.2 & 0.5 & 0.1 \\
$20: 2$ & 3.0 & 2.8 & 2.0 & 4.8 \\
$20: 4$ & 1.6 & 2.2 & 0.7 & 2.1 \\
\hline
\end{tabular}

higher $(33-36 \%)$ than in other younger larvae and eggs of total radioactive fatty acids (Table 5). The fatty acid composition of the lipids of Ascaris eggs of the four stages is represented in Table 8. The eggs of all four stages show a striking quantitative similarity in fatty acid composition. The major saturated fatty acids are palmitate and stearate, and the major unsaturated fatty acids are oleate and linolate. The concentrations of the branched $\mathrm{C}_{15}$ fatty acid, are 1.6 to $2.0 \%$ of the total fatty acid in eggs at all stages of embryonation. This observation is consistent with the results previously mentioned by Jezyk (1968) and Tarr and Fairbairn (1973), who reported $1.9 \%$ and $1.24 \%$ of the fatty acid in ovarian triglyceride of Ascaris lumbricoides, respectively. In aerobic conditions, it was observed that the incorporation of radioactivity in $\mathrm{C}_{18: 1}$ acid was decreased and that in branched $\mathrm{C}_{15}$ fatty acid was greatly increased in the second stage larvae compared with the first stage larvae. In anaerobic conditions, although the production of radioactive $\mathrm{C}_{18: 1}$ acid from acetate- $1-{ }^{14} \mathrm{C}$ was small $(0.8 \%)$, large quantities of branched $\mathrm{C}_{15}$ fatty acid was produced by acetate-1-1 ${ }^{14} \mathrm{C}$ in second stage larvae, similar to aerobic conditions. From these results, it appears that a metabolic change of the fatty acid biosynthesis may occur during development of Ascaris lumbricoides eggs to second stage larvae. We have confirmed that this branched 15 carbon acid is isopentadecanoic acid in the two following experiments. Fig. 2 illustrates the relative retention times of isobranched fatty acids mixtures (Applied Science Lab., Inc.) and this branched 15 carbon acid by gas liquid chromatography. The retention time of the fatty acid is in close agreement 


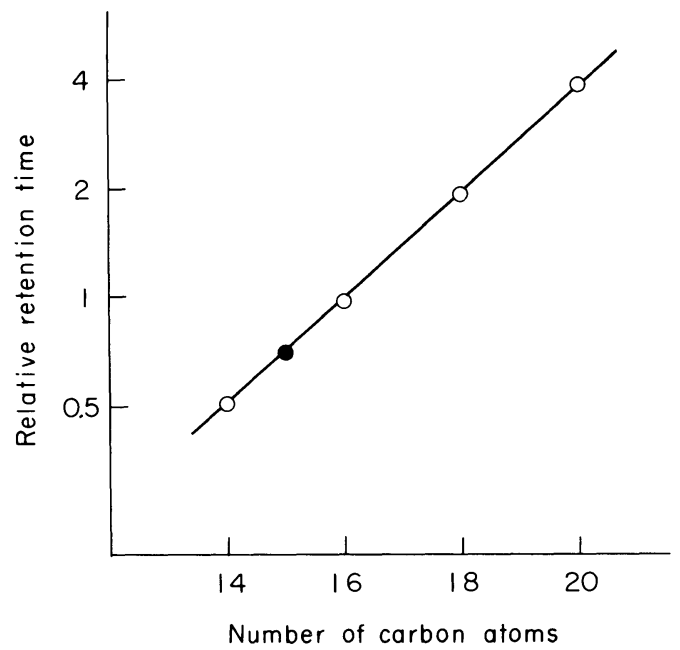

Fig. 2. Retention times of isobranched fatty acids. Open and closed circles represent the relative retention times of standard isobranched fatty acid methylester (Applied Science Lab., Inc.) and unknown fatty acid methylester from Ascaris eggs, respectively. Samples were subjected to gas liquid chromatography (Yanako Gas Chromatograph, Model G 180 equipped with dual hydrogen flame-ionization detector). The apparatus contained a $3 \mathrm{~m} \times$ $3 \mathrm{~mm}$ stainless steel column containing 80-100 mesh chromosorb W coated with $15 \%$ diethylenglycol succinate polyester. Nitrogen was used the carrier gas at a flow rate of $40 \mathrm{ml} / \mathrm{min}$. Temperatures of column, injection port and detector were $195^{\circ} \mathrm{C}, 250^{\circ} \mathrm{C}$ and $250^{\circ} \mathrm{C}$, respectively.

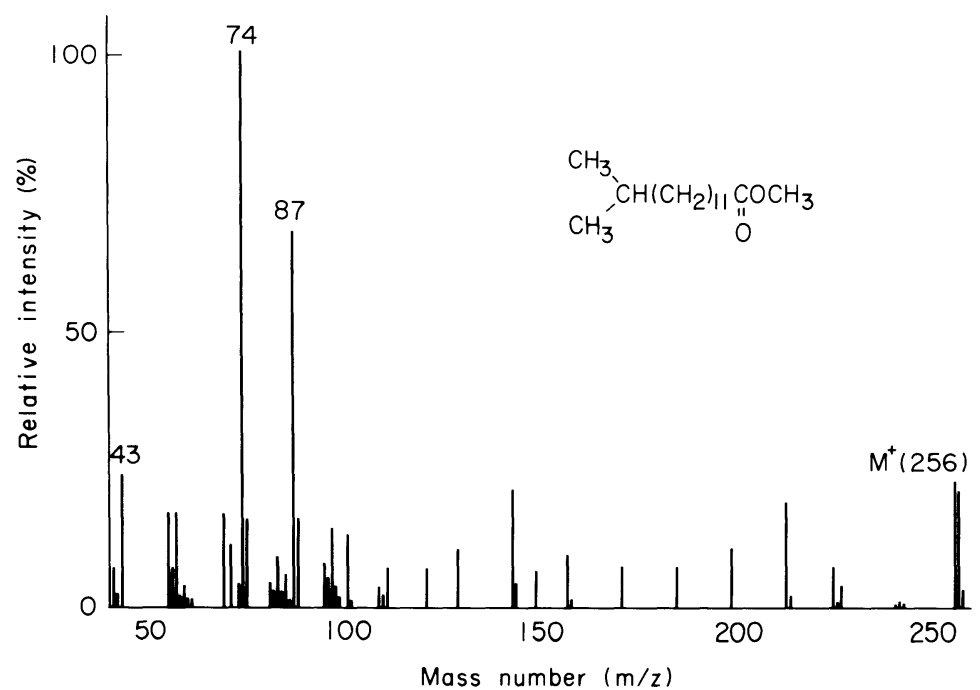

Fig. 3. Mass spectrum of unknown fatty acid methylester. Mass spectrum was obtained on a Hitachi M-52G Mass Spectrometer under ionizing voltage $25 \mathrm{eV}$ and ionizing current $100 \mu \mathrm{A}$. 
with the position of the 15 carbon on the curve of the standard branched fatty acids. The branched fatty acid methylester was purified by a rechromatograph of preparative gas-liquid chromatography as described in materials and methods. The mass spectrum of the branched fatty acid methylester is shown in Fig. 3. The parent peak of this ester was observed at $m / z 256$. Fragment peaks of $m / z$ 87, 74 and 43 were intense enough for easy identification. These peaks can be assigned to the $\left(\mathrm{CH}_{2} \mathrm{CH}_{2} \mathrm{CO} \cdot \mathrm{OCH}_{3}\right),\left(\mathrm{CH}_{2} \mathrm{CO} \cdot \mathrm{OCH}_{3}\right)$ and isopropyl groups, respectively.

\section{Discussion}

Ascaris lumbricoides may synthesize fatty acids de novo by means of the malonyl-CoA pathway, though only at a very low level (Beames et al. 1967). Since the worm does assimilate large amounts of lipids in the process of egg production, it must fall back upon its host diet to provide the required concentration of fatty acids. Since the fat metabolism is catabolic in the eggs and larvae, as suggested by Jazyk and Fairbairn (1967), it may be considered that all fats utilized as energy sources during the development of eggs are provided by the mother worm. We suggested that Ascaris eggs and larvae could not only utilize the fatty acids for development but also biosynthesized certain necessary fatty acids and lipids from these fatty acids (Tables 2 and 3). From investigation of the distribution of radioactivity in the various classes of lipids, it was determined that the conversion to fatty acid methyl esters from palmitate- $1-{ }^{14} \mathrm{C}$ increased with the progress of development of the larvae (Table 3 ). The radioactive fatty acid methyl esters were at the highest concentration in the radioactive lipid classes during the first and second stages of the larvae. Ward and Fairbairn (1970) demonstrated that during development there were changes in the specific activities of many enzymes involved in $\beta$-oxidation and the tricarboxylic acid cycle. The activities of the long chain acyl-CoA synthetase and acyl-CoA dehydrogenase are maximal at 14 days and closely parallel changes in the rates of triglyceride utilization and of palmitic acid-U- ${ }^{14} \mathrm{C}$ oxidation. Costello et al. (1963), who studied the respiration of developing Ascaris eggs, found an absence of significant amounts of cytochrome $\mathrm{C}$ oxidase activity in the initial stage of the egg development, and concluded that FAD was involved as part of the terminal transport system. Contrary to the unembryonated eggs, the second stage larvae, which have the ability to respire, depend upon the cytochrome oxidase. Oya et al. (1963) studied the activity of cytochrome oxidase during development in Ascaris eggs. They indicated that the oxidase activity increased suddenly at the same time in agreement with the period of second stage larvae formation. The increase of acetate- $1-{ }^{14} \mathrm{C}$ incorporation into isopentadecanoic acid in second stage larvae observed in this investigation suggests that there may be a developmental change in fatty acid synthesis in the larvae during embryonation of Ascaris eggs. The fatty acid, isopentadecanoic acid, has been found in only a small percentage (1.9 
$\%$ ) in ovarian triglyceride of Ascaris lumbricoides (Jezyk 1968). In contrast, the free living spirochete Treponema zuelzerae contains a large amount of the iso-15: 0 fatty acid (16.4\%) (Meyer and Meyer 1971). Leishmania denovani or Bdellovidria contains a small percentage of branched-chain fatty acids (Beach et al. 1982 ; Andreev et al. 1983). Beames et al. (1967) reported that acetate- $1-{ }^{14} \mathrm{C}$ was incorporated into the lipids of Ascaris muscle and reproductive tissue and that the saturated 16 carbon acid contained a high percentage of the total radioactivity in both tissue homogenates. However, the radioactivity trapped from the effluent of GLC at the times which correspond with the fatty acid of branched 15 carbon acid was not detected in the ovary-oviduct and detected in small amounts $(0.42 \%)$ in muscle fatty acids. The present results, which show a large amount of radioactivity during the transformation from acetate- $1-{ }^{14} \mathrm{C}$ to isopentadecanoic acid in second stage larvae (Table 5), may indicate a distinctive feature of the fatty acid metabolism in these larvae.

Our preliminary study (Kimura et al. 1977, Table 9) revealed that acetate-1-14 C was also incorporated into the iso-branched fatty acid in adult female Ascaris in vivo. It may be assumed that the parasite interchanges the fatty acid synthesizing system in embryonation and provides the iso-branched fatty acid formation system during the second stage development. Meyer and Holz (1966), who investigated the distribution of radioactivity among saturated fatty acids of

TABLE 9. Major fatty acid composition in adult female of Ascaris lumbricoides and distribution of radioactivity in the fatty acid after injection of acetate-1 ${ }^{14} \mathrm{C}$ into the parasite

\begin{tabular}{rrrrr}
\hline & \multicolumn{2}{c}{$\begin{array}{c}\text { Fatty acid } \\
\text { composition }(\%)\end{array}$} & \multicolumn{2}{c}{$\begin{array}{c}\text { Distribution of } \\
\text { radioactivity }(\%)\end{array}$} \\
\cline { 2 - 5 } Fatty acid & $\begin{array}{c}\text { Neutral } \\
\text { lipid }\end{array}$ & $\begin{array}{c}\text { Phospho- } \\
\text { lipid }\end{array}$ & $\begin{array}{c}\text { Neutral } \\
\text { lipid }\end{array}$ & $\begin{array}{c}\text { Phospho- } \\
\text { lipid }\end{array}$ \\
\hline$<14: 0$ & 0.0 & 0.0 & 10.0 & 2.6 \\
$14: 0$ & 1.2 & 0.4 & 15.2 & 10.5 \\
iso-15 & 2.0 & 1.0 & 30.2 & 33.4 \\
$15: 0$ & 0.6 & 0.4 & 6.5 & 7.3 \\
$16: 0$ & 14.3 & 8.7 & 15.8 & 26.0 \\
$16: 1$ & 1.2 & 0.9 & 3.2 & 5.4 \\
$17: 0$ & 1.7 & 1.0 & 6.1 & 12.3 \\
$17: 1$ & 0.4 & 0.8 & 1.0 & 0.0 \\
$18: 0$ & 25.3 & 23.3 & & 2.2 \\
$18: 1$ & 23.7 & 29.4 & 11.8 & 0.2 \\
$18: 2$ & 21.1 & 15.3 & 0.0 & 0.0 \\
$>18: 2$ & 7.2 & 18.8 & 0.0 & 0.0 \\
\hline
\end{tabular}

Female Ascaris lumbricoides were administrated acetate- $1-{ }^{14} \mathrm{C}(1 \mu \mathrm{Ci})$ by injection into the pseudocoelom, for $12 \mathrm{hr}$ at room temperature, to determine distribution of radioactivity into the fatty acids. 
Crithidia fasciculata grown with isovaleric acid- $1-{ }^{14} \mathrm{C}$ and ${ }^{14} \mathrm{C}$-leucine, indicated a high radioactivity in the fatty acid isopentadecanoic acid. On the other hand, when Ascaris lumbricoides ferments carbohydrate to 2-methylbutylate and 2methyvalerate as the end products, propionyl-CoA may be the direct precursor of the branched chain volatile acids by a pathway similar to a reverse of the $\beta$-oxidation pathway (de Mata et al. 1977). It has been suggested that the synthesis of branched-chain acids may play a role in generation of energy and a regulatory role in maintaining the mitochondrial redox balance (Komuniecki et al. 1981). Furthermore, Horning et al. (1960) reported that $2-{ }^{14} \mathrm{C}$-malonyl-CoA was incorporated into iso- $\mathrm{C}_{15}$ fatty acid in rat adipose tissue enzyme preparation incubated with isovaleryl-CoA as a acyl-CoA accepter. From these results, the formation of the fatty acid may be considered a chain elongation of isovalerylCoA derived from leucine (Horning et al. 1960 ; Law et al. 1963 ; Kates 1964). It is possible that in second stage larvae of Ascaris lumbricoides the fatty acid synthesizing system convert from even numbered long chain fatty acid synthesis to isopentadecanoic acid formation for adaptation to the reduced environment. The definite role of lipid metabolism in isobranched fatty acid synthesis remains to be clarified.

\section{References}

1) Andreev, L.V., Afinogenova, A.V., Penabad, Z.R. \& Lambina, V.A. (1983) Peculiarities of the fatty acid composition of Bdellovibrio. Folia microbiol., 28, 28-35.

2) Barrett, J., Ward, C.W. \& Fairbairn, D. (1970) The glyoxylate cycle and the conversion of triglycerides to carbohydrates in developing eggs of Ascaris lumbricoides. Comp. Biochem. Physiol., 35, 577-586.

3) Beach, D.H., Holz, G.G. Jr., Semprevivo, L.H. \& Honigberg, B.M. (1982) Temperature-dependent fatty acyl group changes in phospholipids of $37 \mathrm{C}$-adapted Leishmania donovani promastigotes. J. Parasitol., 68, 1004-1009.

4) Beames, C.G. Jr., Harris, B.G. \& Hopper, F.A., Jr. (1967) The synthesis of fatty acids from acetate by intact tissue and muscle extract of Ascaris lumbricoides suum. Comp. Biochem. Physiol., 20, 509-521.

5) Costello, L.C., Oya, H. \& Smith, W.N. (1963) The comparative biochemistry of developing Ascaris eggs. I. Substrate oxidation and the cytochrome system in embryonated and unembrionated eggs. Arch. Biochem. Biophys., 103, 345-351.

6) de Mata, Z.S., Saz, H.J. \& Pasto, D.J. (1977) 2-Methylacetoacetate reductase and possible propionyl Coenzyme A condensing enzyme activity in branched chain volatile fatty acid synthesis by Ascaris lumbricoides. J. biol. Chem., 252, 4215-4224.

7) Fairbairn, D. (1955) Embryonic and postembryonic changes in the lipids of Ascaris lumbricoides eggs. Canad. J. Biochem. Physiol., 33, 122-129.

8) Fairbairn, D. (1960) The physiology and biochemistry of nematodes. In: Nematology, edited by J.N. Sasser \& W.R. Jenkins, The University of North Carolina Press, Chapel Hill, North Carolina, pp. 267-296.

9) Horning, M.G., Martin, D.B., Karmen, A. \& Vagelos, P.R. (1960) Synthesis of branched-chain and odd-numbered fatty acids from malonyl-CoA. Biochem. biophys. Res. Comm., 3, 101-106.

10) Jezyk, P.F. (1968) Glycerolipid metabolism in Ascaris lumbricoides. Canad. J. Biochem. Physiol., 46, 1167-1173. 
11) Jezyk, P.F. \& Fairbairn. D. (1967) Ascarosides and ascaroside esters in Ascaris lumbricoides (Nematoda). Comp. Biochem. Physiol., 23, 691-705.

12) Jezyk. P.F. \& Fairbairn, D. (1969) Quantitative aspects of lipid utilization during embryogenesis of Ascaris suum eggs. J. Parasit., 55, 477-479.

13) Kates, M. (1964) Bacterial lipids. Advan. Lipid Res., 2, 17-90.

14) Kimura, S., Furukawa, Y., Aoyama, Y., Yamamoto, T., Oya, H. \& Hayashi, H. (1977) The studies on the desaturation of fatty acids in the pig Ascaris. 20th International Conference on the Biochemistry of Lipids, Aberdeen, Scotland, pp. 13.

15) Komuniecki, R., Komuniecki, P.R. \& Saz, H.J. (1981) Relationships between pyruvate decarboxylation and branched-chain volatile acid synthesis in Ascaris mitochondria. J. Parasit., 67, 601-608.

16) Law, J.H., Zalkin, H. \& Kaneshiro, T. (1963) Transmethylation reactions in bacterial lipids. Biochim. biophys. Acta, 70, 143-151.

17) Metcalfe, L.D. \& Schmitz, A.A. (1961) The rapid preparation of fatty acid esters for gas chromatographic analysis. Anal. Chem., 33, 363-364.

18) Meyer, H. \& Holz, G.G., Jr. (1966) Biosynthesis of lipids by Kinetoplastid flagellates. J. biol. Chem., 241, 5000-5007.

19) Meyer, H. \& Meyer, F. (1971) Lipid metabolism in the parasitic and free-living spirochetes Treponema pallidum (Reiter) and Treponema zuelzerae. Biochim. biophys. Acta, 231, 93-106.

20) Oya, H., Costello, L.C. \& Smith, W.N. (1963) The comparative biochemistry of developing Ascaris eggs. II. Changes in cytochrome C oxydase activity during embryonation. J. cell. comp. Physiol., 62, 287-293.

21) Passey, R.F. \& Fairbairn, D. (1955) The respiration of Ascaris lumbricodies eggs. Canad, J. Biochem. Physiol., 33, 1033-1046.

22) Saz, H.J. \& Lescure, O.L. (1965) ${ }^{14} \mathrm{CO}_{2}$ fixation into free and bound volatile acids of Ascaris lumbricoides eggs. J. Parasit., 51, 56-59.

23) Saz, H.J. \& Lescure, O.L. (1966) Interrelationships between the carbohydrate and lipid metabolism of Ascaris lumbricoides egg and adult stages. Comp. Biochem. Physiol., 18, 845-857.

24) Stumpf, P.K. (1962) Lipid metabolism in higher plants. Nature, 194, 1158-1160.

25) Tarr, G.E. \& Fairbairn, D. (1973) Glycerides, waxes and sterols in ovaries of Ascaris lumbricoides (Nematoda). Lipids, 8, 303-310.

26) Von Brand, T. (1952) Chemical Physiology of Endoparasitic Animals, Academic Press, New York.

27) Wakil, S.J., Porter, J.W. \& Gibson, D.M. (1957) Studies on the mechanism of fatty acid synthesis. I. Preparation and purification of an enzyme system for reconstruction of fatty acid synthesis. Biochim. biophys. Acta, 24, 453-461.

28) Ward, C.W. \& Fairbairn, D. (1970) Enzymes of $\beta$-oxidation and their function during development of Ascaris lumbricoides eggs. Develop. Biol., 22, 366-387. 